Med Principles Pract 1992-93;3:235

\title{
Author Index Vol. 3, 1992-93
}

Abdelkadir, E.A. 213 Abdul Majeed, O. 223 Abu Farsakh, F.A.F.R.

21 Adeloye,A. 185 Al-Adnani, M.S. 51 Al-Awadi, S.A. 156 Albayrak,Y. 60 Al-Fayez, G.A. 72 Al-Harbi, S.A. 199 Al-Kouka,N. 185 Al-Nassar, K.E. 181 Al-Saigh, M.R. 185 Al-Sayed, A.M. 102 Aneas, F.C. 25 Anton, A.Y. 167 Aoki,K. 63 Aritas, Y. 171 Awad El Karim, M.A.

213

Bener,A. 181 Bengisu,N. 171 Berg,B. 118

Chrisikos,N. 17 Christensson, J.T. 77 Chugh,T.D. 44,160

Dashti,H. 77 Dauod,A.S. 57 Dhar,R. 44 Diab,S.M. 51

Eklof,B. 141 El-Badramany, M.H.

156 ElgoharyA.G. 185 Elhag,K.M. 77 El-Khalifa, M.Y. 156 ElSaleh,Q. 223 ElShilla,G. 223 ElTomi,N. 199,227

Farag, S.I. 160 Farag,T.I. 156 Farah, S. 51 Farah,S.A.R. 118 Farid,L. 31 Fido,A.A. 72,195

Fouad,F. 199 Francis, I.M. 31 Furuyama, F. 63

Ghafoor,M.A. 44 Grape,E. 40 Gumaa,K.A. 213

Haddad,J.F. 118 Harrison, A. 88 Hashitani, T. 63 Hassanein, A.A. 57 Hayat,L. 77 Hendow,M.T. 160 Hira,P.R. 31 Huq,E. 44

Isobe, Y. 63

Jamil, A.S. 54 Johny,M. 54,167

Kaaba,S $\Lambda .199$ Karachalios, G.N. 17 Karnik,A.M. 51,115 Kehagioglou, K. 17 Khalid,A. 21

Khogali,M. 213 Kouka,N. 21

Leven, H. 40

Mahmoud,N.A. 213 Makhseed,M. 227 Matusik, J. 31 Menon,N.K. 54 Moody, P.M. 174

Motaweh, M.M. 25 Moussa, M.A.A. 141 Murad,M.A. 167

Naeverdal,T. 40 Naguib,K,K. 156 Nandakumaran, M. 84, 219

Omar,Y. 72 Omarah, Z. 25 Owunwanne, A. 147

Poser, CM. 1

Razik,M.A. 195

Shah, M.A. 102,131 Shah,N.M. 102,131 Shaltout,A.A. 54 Sharma,P.N. 232 Shawa,N. 141

Sherif,M.F. 204 Sigurdsson, G.H. 147 Simsek,M. 181 Sozuer,E.M. 171 Starzl,T. 199 Stormer,J. 40 Sugathan, T.N. 219 Sulaiman, K.J. 115

Teebi,A.S. 156 Temel,S.A. 60 Thulesius,O. 123,141 Tibblin,S. 118 Tsantilas, C. 17 Tsatsa, E. 17 Tungekar, F.M. 31

Vallgren, M. 77 Vallgren,S. 77

Walsh, RJ. 204 Watmough, D.J. 232

Yesilkaya,Y. 171 Yilmaz,Z. 171 Youssef, H.A.E. 25,147

Zaki,M. 57,223 Zend,M.E. 25

235 